\title{
Efficacy of Natalizumab and Fingolimod in Relapsing Remitting Multiple Sclerosis in Real World Clinical Setting
}

Totaro $\mathbf{R}^{1}$, Costantino $\mathbf{G}^{2}$, Bellantonio $\mathbf{P}^{3}$, Danni $\mathbf{M}^{4}$, Di Carmine $\mathbf{C}^{1}$, Fantozzi $\mathbf{R}^{3}$, Cerqua $\mathbf{R}^{4}$, Fuiani $\mathbf{A}^{2}$, Carrocci $\mathbf{C}^{1}$, Mundi $\mathbf{C}^{2}$, Ruggieri $\mathbf{S}^{3}$, Marini $\mathbf{C}^{1}$, Provinciali $\mathrm{L}^{4}$ and Carolei $\mathbf{A}^{1}$

${ }^{1}$ Department of Neurology, University of L'Aquila, L'Aquila, Italy

${ }^{2}$ Department of Neurology, Ospedali Riuniti, Foggia, Italy

${ }^{3}$ Department of Neurology, IRCCS MEUROMED, Pozzilli, Italy

${ }^{4}$ Department of Neurology, University of Ancona, Ancona, Italy

*Corresponding author: Rocco Totaro, Multiple Sclerosis Center, Department of Neurology, University of L'Aquila, 67100 L'Aquila, Tel: +390862368322; E-mail: rocco.totaro@univaq.it

Received date: Oct 16, 2015; Accepted date: Dec 09, 2015; Published date: Dec 16, 2015

Copyright: $@ 2015$ Totaro R, et al. This is an open-access article distributed under the terms of the Creative Commons Attribution License, which permits unrestricted use, distribution, and reproduction in any medium, provided the original author and source are credited.

\begin{abstract}
Objective: The aim of this study was to compare the efficacy of natalizumab and fingolimod in relapsing-remitting multiple sclerosis patients in real-world clinical setting.

Methods: We enrolled 391 patients starting either natalizumab or fingolimod for relapsing-remitting multiple sclerosis, referred to four multiple sclerosis centers between March 2007 and July 2013. Cumulative proportion of patients free from any disease activity, as defined by freedom from relapse, Expanded Disability Status Scale (EDSS) progression, new or newly enlarging T2 lesions, and gadolinium enhancing lesions at magnetic resonance imaging (MRI) was assessed at 12-month follow-up.

Results: Out of 391 patients, 197 were treated with natalizumab and 194 with fingolimod. The cumulative proportion of patients free from any disease activity was $72.0 \%$ in the natalizumab and $59.1 \%$ in the fingolimod group $(\mathrm{P}=0.014)$. This proportion was lower in fingolimod patients with prior natalizumab exposure compared to those without $(51.7 \%$ vs. $61.8 \%$; $P=0.008)$. Moreover, the cumulative proportion of patients free from new MRI lesions was $87.5 \%$ in the natalizumab vs. $70.0 \%$ in the fingolimod group $(P<0.001)$; the cumulative proportion of patients free from clinical relapse was $82.5 \%$ in the natalizumab vs. $81.3 \%$ in the fingolimod group $(P=0.739) ; 93.5 \%$ of patients on natalizumab were free from EDSS progression compared to $89.6 \%$ of patients on fingolimod $(P=0.186)$.
\end{abstract}

Conclusions: Results from 1-year follow-up of treatment suggest higher efficacy of natalizumab compared to fingolimod in terms of proportion of patients free from any disease activity.

Keywords: Multiple sclerosis; Natalizumab; Fingolimod; Postmarketing study; Efficacy

\section{Introduction}

Multiple sclerosis (MS) is a chronic inflammatory disease of the central nervous system and the second leading cause of neurological disability in young adults. During the past decades substantial effort has been made to broaden the MS treatment armamentarium with the approval of second line drugs effective in breakthrough disease.

To date, second line treatment options available include fingolimod, natalizumab and, to lesser extent, mitoxantrone. Both natalizumab and fingolimod provide a significant control in highly active disease.

Natalizumab is a a4-integrin antagonist that blocks adhesion and diapedesis of leukocytes into the CNS. Two pivotal trials, the AFFIRM (natalizumab vs. placebo) and SENTINEL (natalizumab + interferon beta-1a vs. interferon beta-1a alone) studies proved natalizumab to be effective as monotherapy as well as add-on therapy with first line agents, leading to its final approval as second line treatment for relapsing remitting multiple sclerosis (RRMS) [1,2]. Nevertheless, the risk of progressive multiple leukoencephalopathy raised concern about potential safety issues of natalizumab in so-called "triple risk" patients (previous immunosuppressive agents, positive JCV serology, two-year treatment duration) [3]

Fingolimod is the first oral agent approved for the treatment of multiple sclerosis. Fingolimod acts as a sphingosine 1 phosphate receptor modulator and exerts its mechanism of action preventing lymphocyte egress from lymphoid tissues. Two phase III studies, TRANSFORM and FREEDOMS trials, demonstrated fingolimod as an highly effective treatment in lowering relapse rate and disability progression in relapsing-remitting multiple sclerosis $[4,5]$

Adverse events associated with its use are first dose symptomatic bradycardia and atrio-ventricular block, macular oedema, hypertension and malignancy.

European Medical Agency (EMA) eligibility criteria for either fingolimod and natalizumab include highly active disease despite firstline therapies or breakthrough disease at onset, thus prompting a careful balancing between potential risks and efficacy profiles. 
Page 2 of 6

To date, few observational studies compared efficacy between natalizumab and fingolimod in real clinical setting, while there are still no head-to-head trial comparing efficacy of these drugs, which would eventually improve clinical decision making after switching to second line therapies.

The aim of our study was to assess the efficacy at 1-year follow-up in a cohort of patients treated with either fingolimod or natalizumab in real world clinical practice.

\section{Materials and Methods}

We report data from 391 patients, diagnosed with RRMS according to McDonald Criteria, collected routinely in four multiple sclerosis centers throughout Central and Southern Italy. All patients starting either natalizumab or fingolimod between March 2007 and July 2013 were included. All patients had at least 1-year follow-up period of observation.

Patients were included in accordance to Italian Drug Agency (AIFA) eligibility criteria for reimbursement of the drug. Natalizumab eligibility criteria included: patients on previous immunomodulant treatment for at least 12 months who had experienced either two relapses in the last year or a single relapse with incomplete recovery and residual disability (EDSS $\geq 2.0$ ), with at least 9 T2 lesions at MRI, or an increased lesion burden, or at least 1 gadolinium-enhanced lesion; patients with severe MS with a fast evolution, even if not previously treated with immunomodulant treatments, with $\geq 2$ relapses with increased disability during the last year (EDSS $\geq 2.0$ ), and with new T2 or gadolinium-enhanced lesions on MRI, compared with a previous MRI examination performed during the previous 12 months.

Fingolimod eligibility criteria included: patients on previous immunomodulant treatment for at least 12 months who have experienced at least one relapse in the last year with at least $9 \mathrm{~T} 2$ lesions at MRI, or an increased lesion burden, or at least 1 gadoliniumenhanced lesion; patients with severe MS with a fast evolution, even if not previously treated with immunomodulant treatments, with $\geq 2$ relapses with accumulation of disability during the last year, and with new T2 or gadolinium-enhanced lesions on MRI, compared with a previous MRI examination conducted during the previous 12 months.

Patients switching from natalizumab to fingolimod were also included and started treatment after a wash-out period of three months. All these patients discontinued natalizumab due to safety concerns for positive JCV status and overall treatment duration longer than 24 months, despite being clinically and radiologically stable under natalizumab treatment.

Patients treated with fingolimod underwent complete cardiac evaluation before starting fingolimod in order to exclude heart rate and atrio-ventricular conduction abnormalities. Moreover, a continuous electrocardiographic monitoring for at least six hours after the administration of the first dose was performed in all patients. Every hour, arterial pressure values were also recorded. Ophthalmologic evaluation with optical coherence tomography was performed at baseline, at 3,6, and 12 months, in order to monitor potential adverse events such as macular oedema. Neurological examination and EDSS assessment were performed at baseline and during follow-up visits. EDSS was registered at baseline, at 6 and 12 months. Serious adverse events were recorded at each scheduled visit or whenever they occurred.
MRI scans were obtained at baseline and 12 months. Imaging data were collected at MRI facilities of the participating sites and reviewed by experienced neuroradiologists. MRI imaging was performed at $1.5 \mathrm{~T}$ and at least axial FLAIR, T2 weighted and T1 gadolinium enhanced sequences were obtained for all exams. MRI scans were analyzed for new or newly enlarging T2 lesions and gadolinium enhancing lesions.

Patients were treated with fingolimod $0.5 \mathrm{mg}$ once daily or natalizumab $300 \mathrm{mg}$ every 28 days.

The study was approved by the Azienda Sanitaria Locale AvezzanoSulmona-L'Aquila Ethics Committee.

The main end point was the proportion of patients free from any disease activity as defined by freedom from clinical relapses; from confirmed EDSS progression, as defined by sustained increase of $\geq 1.0$ point in the EDSS score (or $\geq 1.5$ points if the baseline was $<1.0$ ) confirmed after 12 weeks; from new or newly enlarging T2 lesions and/or gadolinium enhancing lesions.

As additional end-points, we also considered each single disease activity measure independently and ARR variations at 6 and 12 months with respect to values in the year prior the start of treatments. In the fingolimod group previously treated with natalizumab the ARR of year preceding treatment start was assumed equal to that in the year prior the natalizumab start.

\section{Statistical analysis}

Continuous variables were reported as means, standard deviations and range while discrete data were reported in contingency tables as absolute and relative frequencies.

The comparisons between groups (fingolimod vs natalizumab and fingolimod previously treated with natalizumab vs fingolimod not previously treated with natalizumab vs natalizumab) were performed using negative binomial regression and Paired Samples t-test. Multivariate analysis was carried out by negative binomial regression model corrected by covariates. Time to first relapse, time to first EDSS worsening and time to first new MRI lesions were evaluated using Kaplan-Meier analysis and curves. Groups (fingolimod vs natalizumab and fingolimod previously treated with natalizumab vs fingolimod not previously treated with natalizumab vs natalizumab) were compared using the log-rank test. Multivariate analysis of survival was carried out by Cox Regression model corrected by covariates.

\begin{tabular}{|l|l|l|l|l|}
\hline & $\begin{array}{l}\text { Natalizumab } \\
(\mathrm{N}=197)\end{array}$ & $\begin{array}{l}\text { Fingolimod } \\
(\mathrm{N}=194)\end{array}$ & $\begin{array}{l}\mathrm{p}- \\
\text { value }\end{array}$ & Total \\
\hline Women (n,\%) & $134(68.0 \%)$ & $130(67.0 \%)$ & 0.831 & $67.50 \%$ \\
\hline Age & $34.5 \pm 9.39$ & $38.9 \pm 9.34$ & $<0.001$ & $36.7 \pm 9.61$ \\
\hline Duration of disease & $9.0 \pm 7.00$ & $10.1 \pm 7.32$ & 0.121 & $9.6 \pm 7.17$ \\
\hline $\begin{array}{l}\text { EDSS in the year } \\
\text { preceding treatment }\end{array}$ & $2.3 \pm 0.95$ & $2.5 \pm 1.14$ & 0.099 & $2.43 \pm 1.05$ \\
\hline $\begin{array}{l}\text { ARR in the year } \\
\text { preceding treatment }\end{array}$ & $1.9 \pm 0.72$ & $1.1 \pm 0.74$ & $<0.001$ & $1.5 \pm 0.84$ \\
\hline $\begin{array}{l}\text { Patients with MRI activity } \\
\text { at baseline (n,\%) }\end{array}$ & $190(96.4 \%)$ & $135(70.7 \%)$ & $<0.001$ & $325(83.8 \%)$ \\
\hline
\end{tabular}

Table 1: Demographic and clinical characteristics of study population (Data are mean \pm SD unless otherwise indicated, EDSS-Expanded Disability Status Scale; ARR-Annualized relapse rate) 
Citation: Totaro R, Costantino G, Bellantonio P, Danni M, Di Carmine C, et al. (2015) Efficacy of Natalizumab and Fingolimod in Relapsing Remitting Multiple Sclerosis in Real World Clinical Setting . J Neurol Neurophysiol 6: 337. doi:10.4172/2155-9562.1000337

Page 3 of 6

A significance level of 0.05 was used for each test. For all the analysis, IBM SPSS Statistics for Windows, Version 20.0, was used.

\section{Results}

Out of 391 patients included, 265 patients were women and 127 men. One-hundred-ninety-seven patients were treated with natalizumab and 194 with fingolimod. Out of 194 patients who started with fingolimod, 52 were previously treated with natalizumab.

Complete demographic and clinical characteristic were reported in the Table 1. As shown in the table, patients who started natalizumab were significantly younger $(34.5 \pm 9.39$ years vs. $38.9 \pm 9.34$ years; $\mathrm{P}<0.001)$, had a higher annualized relapse rate (ARR) $(1.9 \pm 0.72$ vs. $1.1 \pm 0.74 ; \mathrm{P}<0.001)$, and a higher proportion of patients with disease activity at MRI $(96.4 \%$ vs. $70.7 \%$; $\mathrm{P}<0.001)$ compared to patients who started fingolimod.

Table 2 shows cumulative proportion of patients free from disease activity in natalizumab and fingolimod groups at 1-year follow-up.

\begin{tabular}{|c|c|c|c|c|c|c|}
\hline & \multirow[t]{2}{*}{ Natalizumab } & \multirow[t]{2}{*}{ Fingolimod } & \multirow[t]{2}{*}{$P^{*}$} & \multicolumn{2}{|l|}{ Fingolimod } & \multirow[t]{2}{*}{$P^{* *}$} \\
\hline & & & & $\begin{array}{l}\text { Without prior } \\
\text { natalizumab } \\
\text { exposure }\end{array}$ & $\begin{array}{l}\text { With prior natalizumab } \\
\text { exposure }\end{array}$ & \\
\hline $\begin{array}{l}\text { Proportion of patients free from any } \\
\text { disease activity }\end{array}$ & $72.0 \%$ & $59.1 \%$ & 0.014 & $61.8 \%$ & $51.7 \%$ & 0.008 \\
\hline $\begin{array}{l}\text { Proportion of patients free from } \\
\text { MRI lesions }\end{array}$ & $87.5 \%$ & $70.0 \%$ & $<0.001$ & $74.5 \%$ & $68.3 \%$ & $<0.001$ \\
\hline $\begin{array}{l}\text { Proportion of patients free from } \\
\text { relapse }\end{array}$ & $82.5 \%$ & $81.3 \%$ & 0.739 & $86.3 \%$ & $68.2 \%$ & 0.01 \\
\hline $\begin{array}{l}\text { Proportion of patients free from } \\
\text { EDSS progression }\end{array}$ & $93.5 \%$ & $89.6 \%$ & 0.186 & $92.2 \%$ & $82.5 \%$ & 0.06 \\
\hline
\end{tabular}

Table 2: Cumulative proportion of patients free from disease activity at 1-year follow-up $\left({ }^{*}\right.$ Comparison between natalizumab and fingolimod groups; ${ }^{*}$ Comparison between natalizumab, fingolimod without prior natalizumab exposure, and fingolimod with prior natalizumab exposure; EDSS-Expanded Disability Status Scale; MRI-Magnetic Resonance Imaging)

In detail, the cumulative proportion of patients free from any disease activity was $72.0 \%$ in natalizumab and $59.1 \%$ in fingolimod group (long rank test; $\mathrm{P}=0.014$ ). This proportion was $51.7 \%$ in the group of patients treated with fingolimod and previously treated with natalizumab whereas the proportion was $61.8 \%$ in the fingolimod group not previously treated with natalizumab (long rank test; $\mathrm{P}=0.008$ ) (Figure 1).

Cox regression analysis showed that patients in the fingolimod group previously treated with natalizumab had a greater risk of disease activity (HR 2.15, 95\%CI 1.15-4.01, $\mathrm{P}=0.016$ ).

The cumulative proportion of patients free from new MRI lesions was $87.5 \%$ in natalizumab and $70.0 \%$ in fingolimod group (long rank test; $\mathrm{P}<0.001)$. This proportion was $74.5 \%$ in the group of patients treated with fingolimod and previously treated with natalizumab whereas the proportion was $68.3 \%$ in the fingolimod group not previously treated with natalizumab (long rank test; $\mathrm{P}<0.001$ ).

Cox regression analysis showed that patients treated with fingolimod previously treated with natalizumab had a greater risk of new MRI lesions than patients treated with natalizumab (HR 0.42, 95\%CI 0.23-0.74, $\mathrm{P}=0.005$ ).

The cumulative proportion of patients free from clinical relapse was $82.5 \%$ in natalizumab group and $81.3 \%$ in fingolimod group (long rank test; $\mathrm{P}=0.739$ ).

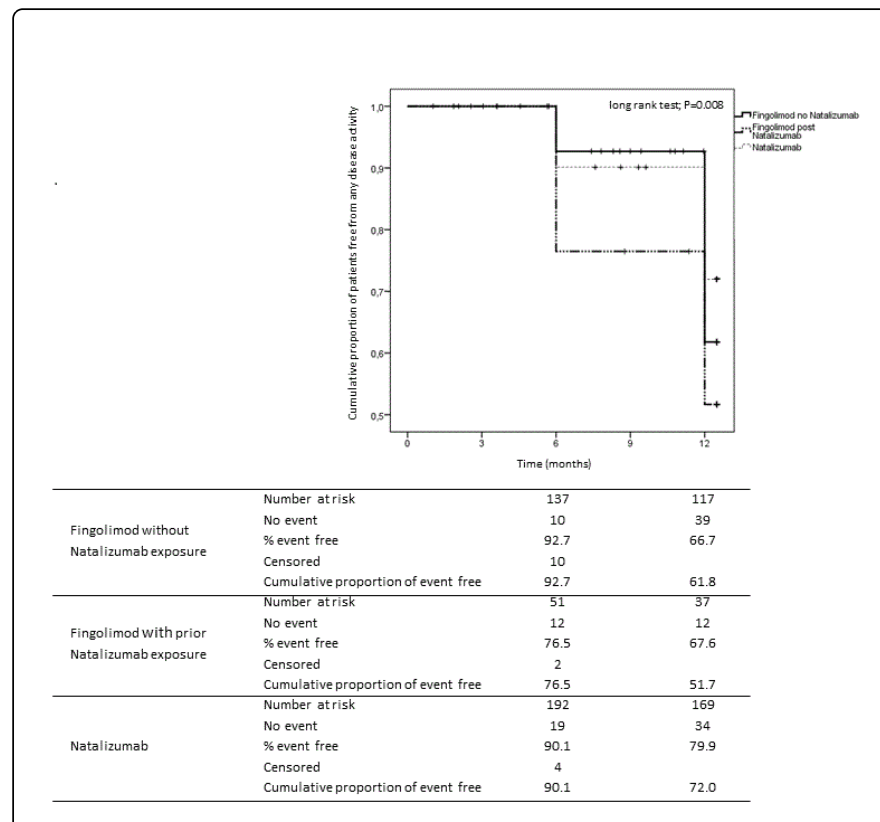

Figure 1: Kaplan-Meyer plot of cumulative proportion of patients remaining free from any disease activity at 1-year follow-up.

This proportion was $68.2 \%$ in the group of patients treated with fingolimod and previously treated with natalizumab whereas the 
proportion was $86.3 \%$ in the fingolimod group not previously treated with natalizumab (long rank test; $\mathrm{P}=0.011$ ).

Multivariate regression analysis showed that both at 6 and 12 months of follow-up the risk of new relapse was significantly associated with the ARR ( $\mathrm{P}=0.001$ and $\mathrm{P}=0.015$, respectively) and the MRI activity $(\mathrm{P}=0.0001$ and $\mathrm{P}=0.0001$, respectively) in the year preceding the start of treatment.

Cox regression analysis showed that patients who started fingolimod after treatment with natalizumab had a greater risk of new relapses with respect to patients treated with fingolimod not previously treated with natalizumab (HR 3.28, 95\% IC 1.47-7.34, P=0.004) and patients treated with natalizumab (HR 2.83, 95\%CI 1.27-6.27, $\mathrm{P}=0.011)$.

Finally, the cumulative proportion of patients free from EDSS progression was $93.5 \%$ in natalizumab group and $89.6 \%$ in fingolimod group (long rank test; $\mathrm{P}=0.186$ ). This proportion was $82.5 \%$ in the fingolimod group with prior natalizumab exposure whereas the proportion was $92.2 \%$ in the fingolimod group not previously treated with natalizumab (long rank test; $\mathrm{P}=0.060$ ).

Multivariate regression analysis showed that both at 6 and 12 months of follow-up the risk of EDSS progression was significantly associated with the number of previous disease modifying treatments ( $\mathrm{P}=0.013$ and $\mathrm{P}=0.021$, respectively).

Cox regression analysis showed that the number of previous disease modifying treatments (HR 2.00, 95\%CI 1.26-3.17, $\mathrm{P}=0.003$ ) and age (HR 1.04, 95\%CI 1.00-1.09, $\mathrm{P}=0.039$ ) were significantly associated with EDSS progression.

ARR significantly lowered after treatment with natalizumab or fingolimod. In the natalizumab group values decreased from 1.88 in the year prior treatment start to 0.20 at 6 months $(\mathrm{P}<0.001)$ and 0.21 at one year $(\mathrm{P}<0.001)$ of treatment. In the fingolimod group values decreased from 1.06 to 0.30 at 6 months $(\mathrm{P}<0.001)$ and 0.23 at one year $(\mathrm{P}<0.001)$ of treatment. In the fingolimod group not previously treated with natalizumab ARR decreased from 1.13 to $0.18(\mathrm{P}<0.001)$ at 6 months and 0.14 at one year $(\mathrm{P}<0.001)$. In the fingolimod group previously treated with natalizumab ARR decreased from 0.85 to 0.62 $(\mathrm{P}=0.308)$ at 6 months and 0.46 at one year $(\mathrm{P}=0.038)$.

No PML cases or other serious adverse events were reported during the 12-month study period.

\section{Discussion}

Over the past two decades, treatment options for relapsing remitting multiple sclerosis have broadened out hugely. Fingolimod and natalizumab have both proven effective in relapsing remitting multiple sclerosis thus being offered as second-line treatments after failure of prior immunomodulatory drugs or in breakthrough disease.

Phase III trials comparing natalizumab and fingolimod to placebo were the AFFIRM and FREEDOMS trial, respectively. In the AFFIRM trial natalizumab reduced the ARR by $68 \%$, lesion burden accumulation by $83 \%$ and the risk of sustained disability progression by $42 \% .1$ In the FREEDOMS trial fingolimod reduced the ARR by $54 \%$, accumulation of new or newly enlarging lesions by $74 \%$ and the risk of sustained disability progression by $30 \% .5$ Yet, evidence coming from head-to-head trials is still lacking. Thus far, direct comparative data still come from few observational studies trying to address their relative efficacy in real world setting. Thus, the purpose of this study was to evaluate the 1-year follow-up clinical and radiological outcomes in a cohort of patients prescribed with either fingolimod or natalizumab.

Baseline characteristics of the study population were similar between groups except for baseline disease activity as defined by MRI activity and ARR in the year preceding study enrolment. Different enrolment times can partly account for higher disease activity in the natalizumab group since many patients were enrolled before 2011 when fingolimod was not yet available. Besides, therapeutic trend shifted towards increasingly early treatment switch in unresponsive disease thus providing patients with lower disease activity with second line treatment options.

In our study a significant greater proportion of patients receiving natalizumab remained free from any disease activity compared with fingolimod treated patients. Higher efficacy was largely driven by a greater impact of natalizumab on radiological disease activity measures, namely lesion burden and gadolinium enhancing lesions. The potent anti-inflammatory effect of natalizumab and the sensitivity of MRI in detecting inflammatory changes in active MS may partly account for these differences.

Even if the proportion of patients free from EDSS progression and clinical relapse did not significantly differ between groups, except for a higher relapse risk in the fingolimod group with prior natalizumab exposure, longer follow-up is needed to ascertain clinical benefit of natalizumab treatment over time.

Both groups had a similar proportion of patients with stable EDSS and subgroup analysis in the fingolimod group did not show any statistically significant difference regardless prior natalizumab exposure. Of further note, the only independent factor associated with EDSS progression was the number of previous disease modifying treatments. This finding backs the hypothesis of longer disease duration and higher disease activity as the underlying cause of disability progression despite second-line therapy. The lack of an outstanding greater efficacy of natalizumab treatment on EDSS in not surprising since the beneficial effect on disability progression lags behind its impact on MRI measures of disease activity. Therefore only prospective, longitudinal studies might disclose relative efficacies of both natalizumab and fingolimod in halting disability progression over long periods of time.

So far, data from literature failed to prove compelling evidence of superiority of natalizumab over fingolimod or vice versa [6]. Recently observational studies showed lower relapse risk and new gadolinium enhancing lesions in natalizumab treated patients vs. fingolimod group, with a follow-up ranging from 18 to 20 months [7-9]. Yet, a study conducted in a German outpatient cohort revealed similar clinical efficacy in either cohorts in terms of EDSS, relapse free and progression free probability [10]. Furthermore, several post-marketing studies have attempted to address efficacy of fingolimod or natalizumab in real clinical setting [11-24]. Hersh et al. reported clinical practice data from a cohort of patients prescribed with fingolimod from 2010 to 2011 with a mean follow-up of 12 months [25]. Compared to our cohort of patients on fingolimod, freedom from relapse or new gadolinium enhancing lesions was achieved in a higher proportion of patients. Many possible confounding factors can undermine this difference such as selection bias. Following the Food and Drug Administration (FDA) fingolimod approval as first line treatment for RRMS, in the aforementioned study only a small proportion of patients were prescribed with fingolimod for 
breakthrough disease $(24.9 \%)$ whereas the majority switched because of patient preference $(47.6 \%)$ thus reflecting a higher disease activity in our population.

We did not find statistically significant difference between groups in terms of cumulative proportion of patients free from clinical relapse. Nevertheless, the subgroup analysis in the fingolimod cohort disclosed a significant difference in the proportion of patients being relapse free among those without natalizumab prior treatment as compared with those switching from natalizumab to fingolimod $(86.3 \%$ and $68.2 \%$, respectively) and with the natalizumab group as well (82.5\%).

Studies have also assessed the outcomes of patients switching from natalizumab to fingolimod in real world setting [26-31]. The expected higher relapse risk up to 6 months after therapeutic switch is largely borne out by results of many observational studies, the higher risk being related to longer wash out period (2-4 months) and higher number of relapse in the previous 6 months [29]. Some authors argued that ARR in patients with prior natalizumab exposure drops after 12 months of treatment with no statistically significant difference with fingolimod naïve patients. One could infer that higher relapse risk up to 6 months after therapeutic shift is underpinned by rebound of disease activity after natalizumab withdrawal and that a shorter wash out period up to 8 weeks would be advisable to prevent disease recurrence early after fingolimod start [32]. In our cohort $31.8 \%$ of fingolimod patients with prior natalizumab exposure relapsed at 1year follow-up. This result is consistent with findings from previous literature reporting clinical relapses after therapeutic switch ranging from $20 \%$, at 6 months, up to $41 \%$, at 10 months [26,27]. The aim of this study was to provide an insight from real life efficacy data. To this end we included in the analysis both fingolimod naïve patients and those with prior natalizumab exposure. Nevertheless, direct comparison between natalizumab group and fingolimod naïve patients did not show any statistically significant difference in terms of relapse risk, EDSS progression. Yet, natalizumab proved to be more efficacious in avoiding new MRI lesions as compared to fingolimod. The cumulative proportion of patients free from new MRI lesions was still lower in the fingolimod group when analysed for possible confounder such as prior natalizumab exposure therefore prompting a higher natalizumab efficacy on radiological measures.

In conclusions, results from the present observational study suggest greater efficacy of natalizumab over fingolimod in a real-world setting. In our study, a higher proportion of patients treated with natalizumab showed absence of combined clinical and radiological disease activity being largely driven by radiological outcome parameters. These results are not at odds with previous findings from literature. Since direct comparison coming from head-to-head studies is still lacking, longterm follow-up real world studies are warranted to attempt filling this gap.

\section{References}

1. Polman $\mathrm{CH}$, O'Connor PW, Havrdova E, Hutchinson $\mathrm{M}$, Kappos L, et al. (2006) A randomized, placebo-controlled trial of natalizumab for relapsing multiple sclerosis. N Engl J Med 354: 899-910.

2. Rudick RA, Stuart WH, Calabresi PA, Confavreux C, Galetta SL, et al. (2006) Natalizumab plus interferon beta-1a for relapsing multiple sclerosis. N Engl J Med 354: 911-923.

3. Kappos L, Bates D, Edan G, Eraksoy M, Garcia-Merino A, et al. (2011) Natalizumab treatment for multiple sclerosis: updated recommendations for patient selection and monitoring. Lancet Neurol 10: 745-758.
4. Cohen JA, Barkhof F, Comi G, Hartung HP, Khatri BO, et al. (2010) Oral fingolimod or intramuscular interferon for relapsing multiple sclerosis. $\mathrm{N}$ Engl J Med 362: 402-415.

5. Kappos L, Radue EW, O'Connor P, Polman C, Hohlfeld R, et al. (2010) A placebo-controlled trial of oral fingolimod in relapsing multiple sclerosis. N Engl J Med 362: 387-401.

6. Bergvall N, Lahoz R, Reynolds T, Korn JR (2014) Healthcare resource use and relapses with fingolimod versus natalizumab for treating multiple sclerosis: a retrospective US claims database analysis. Curr Med Res Opin 30: 1461-1471.

7. Carruthers RL, Rotstein DL, Healy BC (2014) An observational comparison of natalizumab vs. fingolimod using JCV serology to determine therapy. Mult Scler 20: 1381-1390.

8. Gajofatto A, Bianchi MR, Deotto L, Benedetti MD (2014) Are natalizumab and fingolimod analogous second-line options for the treatment of relapsing-remitting multiple sclerosis? A clinical practice observational study. Eur Neurol 72: 173-180.

9. Kalincik T, Horakova D, Spelman T, Jokubaitis V, Trojano M, et al. (2015) Switch to natalizumab versus fingolimod in active relapsing-remitting multiple sclerosis. Ann Neurol 77: 425-435.

10. Braune S, Lang M, Bergmann A; NTC Study Group (2013) Second line use of Fingolimod is as effective as Natalizumab in a German out-patient RRMS-cohort. J Neurol 260: 2981-2985.

11. Butzkueven H, Kappos L, Pellegrini F, Trojano M, Wiendl H, et al. (2014) Efficacy and safety of natalizumab in multiple sclerosis: interim observational programme results. J Neurol Neurosurg Psychiatry 85: 1190-1197.

12. van Pesch V, Bartholomé E, Bissay V (2014) Safety and efficacy of natalizumab in Belgian multiple sclerosis patients: subgroup analysis of the natalizumab observational program. Acta Neurol Belg 114: 167-178.

13. Fernández O, Oreja-Guevara C, Arroyo R, Izquierdo G, Pérez JL, et al. (2012) Natalizumab treatment of multiple sclerosis in Spain: results of an extensive observational study. J Neurol 259: 1814-1823.

14. Melin A, Outteryck O, Collongues N (2012) Effect of natalizumab on clinical and radiological disease activity in a French cohort of patients with relapsing-remitting multiple sclerosis. J Neurol 259: 1215-1221.

15. Kallweit U, Jelcic I, Braun N (2012) Sustained efficacy of natalizumab in the treatment of relapsing-remitting multiple sclerosis independent of disease activity and disability at baseline: real-life data from a Swiss cohort. Clin Neuropharmacol 35: 77-80.

16. Holmén C, Piehl F, Hillert J, Fogdell-Hahn A, Lundkvist M, et al. (2011) A Swedish national post-marketing surveillance study of natalizumab treatment in multiple sclerosis. Mult Scler 17: 708-719.

17. Krysko KM, O'Connor PW (2011) The toronto observational study of natalizumab in multiple sclerosis. Can J Neurol Sci 38: 422-428.

18. Kaufman MD, Lee R, Norton HJ (2011) Course of relapsing-remitting multiple sclerosis before, during and after natalizumab. Mult Scler 17: 490-494.

19. Prosperini L, Borriello G, Fubelli F, Marinelli F, Pozzilli C (2011) Natalizumab treatment in multiple sclerosis: the experience of S. Andrea MS Centre in Rome. Neurol Sci 31 Suppl 3: 303-307.

20. Sangalli F, Moiola L, Bucello S, Annovazzi P, Rizzo A, et al. (2011) Efficacy and tolerability of natalizumab in relapsing-remitting multiple sclerosis patients: a post-marketing observational study. Neurol Sci 31 Suppl 3: 299-302.

21. Outteryck O, Ongagna JC, Zéphir H, Fleury MC, Lacour A, et al. (2010) Demographic and clinic characteristics of French patients treated with natalizumab in clinical practice. J Neurol 257: 207-211.

22. Putzki N, Yaldizli O, Mäurer M (2010) Efficacy of natalizumab in second line therapy of relapsing-remitting multiple sclerosis: results from a multi-center study in German speaking countries. Eur J Neurol 17: 31-37.

23. Putzki N, Yaldizli O, Bühler R (2010) Natalizumab reduces clinical and MRI activity in multiple sclerosis patients with high disease activity: results from a multicenter study in Switzerland. Eur Neurol 63: 101-106. 
Citation: Totaro R, Costantino G, Bellantonio P, Danni M, Di Carmine C, et al. (2015) Efficacy of Natalizumab and Fingolimod in Relapsing Remitting Multiple Sclerosis in Real World Clinical Setting . J Neurol Neurophysiol 6: 337. doi:10.4172/2155-9562.1000337

Page 6 of 6

24. Totaro R, Lugaresi A, Bellantonio P, Danni M, Costantino G, et al. (2014) Natalizumab treatment in multiple sclerosis patients: a multicenter experience in clinical practice in Italy. Int J Immunopathol Pharmacol 27: 147-154.

25. Hersh CM, Hara-Cleaver C, Rudick RA, Cohen JA, Bermel RA et al. (2015) Experience with fingolimod in clinical practice. Int J Neurosci 125 678-85.

26. Bianco A, Patanella AK, Nociti V (2015) Second-line therapy with fingolimod for relapsing-remitting multiple sclerosis in clinical practice: the effect of previous exposure to natalizumab. Eur Neurol 73: 57-65.

27. Baldi E, Guareschi A, Vitetta F (2014) Previous treatment influences fingolimod efficacy in relapsing-remitting multiple sclerosis: results from an observational study. Curr Med Res Opin 30: 1849-1855.

28. Cohen M, Maillart E, Tourbah A, De Sèze J, Vukusic S, et al. (2014) Switching from natalizumab to fingolimod in multiple sclerosis: a French prospective study. JAMA Neurol 71: 436-441.
29. Jokubaitis VG, Li V, Kalincik T, Izquierdo G, Hodgkinson S, et al. (2014) Fingolimod after natalizumab and the risk of short-term relapse. Neurology 82: 1204-1211.

30. Havla J, Tackenberg B, Hellwig K, Meinl I, Krumbholz M, et al. (2013) Fingolimod reduces recurrence of disease activity after natalizumab withdrawal in multiple sclerosis. J Neurol 260: 1382-1387.

31. Rinaldi F, Seppi D, Calabrese M (2012) Switching therapy from natalizumab to fingolimod in relapsing-remitting multiple sclerosis: clinical and magnetic resonance imaging findings. Mult Scler 18: 1640-1643.

32. Kappos L, Radue EW, Comi G, Montalban X, Butzkueven H, et al. (2015) Switching from natalizumab to fingolimod: A randomized, placebocontrolled study in RRMS. Neurology 85: 29-39. 